\title{
Evaluation of a Root Mean Squared Based Ischemia Detector on the Long-Term ST Database with Body Position Change Cancellation
}

\author{
A Mincholé ${ }^{1}$, B Skarp ${ }^{1}$, F Jager $^{2}$, P Laguna $^{1}$ \\ ${ }^{1}$ Comm Techn Group, Aragon Institute of Eng Research, University of Zaragoza, Spain \\ ${ }^{2}$ Faculty of Computer and Information Science, University of Ljubljana, Ljubljana, Slovenia
}

\begin{abstract}
In this work we revisit an ischemia detector based on the root mean square (RMS) series of the repolarization interval developed and validated using the European Society of cardiology ST-T database (ESCDB). This detector, developed within this database framework, gets sensitivity $(S) /$ positive predictivity $(+P)$ performance figures of $85 \% / 86 \%$. Our aim now is to re-evaluate the detector in the much richer Long-Term ST Database where ST episodes of different origin are present, making a much more challenging scenario for the detector. Just a straight forward adaptation of the RMS detector reduces its performance figures, $S /+P$, to $70 \% / 68 \%$. This, apart from other reasons, is a consequence of the presence in the database of $S T$ episodes generated by body position changes (BPC) which can be misinterpreted. A BPC detector incorporated to the previous detector noticeably improves the figures up to $75 \% / 71 \%$.
\end{abstract}

\section{Introduction}

Today 24-hour ECG monitoring is widely used to evaluate patients with suspected or known coronary artery disease. The long duration and huge amount of data for these recordings require some kind of automatization by signal processing techniques in order to effectively detect potential ischemic events on the ECG-signal.

The newly released Long Term ST Database (LTSTDB) [1] contains 24 hour duration records with a large number of human annotated ischemic and non-ischemic STsegment events such as heart rate related events, body position changes or conduction changes giving a much more extensive tool to evaluate and develop ischemia detectors than the early European Society of cardiology ST-T database (ESCDB) [2].

By analysing changes in the ST-segment (see Figure 1), silent ischaemia can often be diagnosed with the help of Holter recordings. However ST segment changes can also result from other causes such as heart rate related events, changes in the electrical axis of the heart due to body position changes (BPC) and conduction changes among others. Reliable ST detectors should distinguish between ischemic and non-ischemic ST changes, although this task remains being a challenge [3].

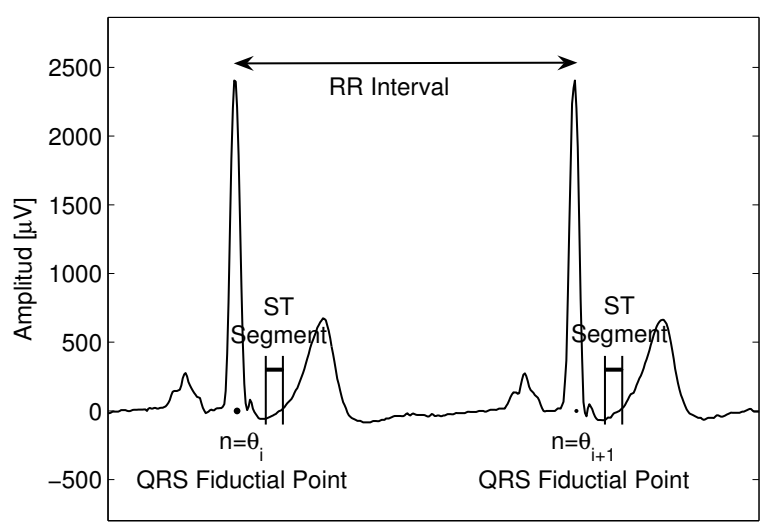

Figure 1. Example of two beats where the QRS fiductial points, the segmented ST length and the RR interval are represented.

Here we will group together heart rate related and ischemic events as the target "event". Therefore we will try to cancel false detections due to BPC and conduction changes. These two kinds of events are responsible for sudden shifts of the ST segment which result in a step function feature in different ECG representations such as Karhunen-Loève Transform (KLT) of the QRS complex and the ST segment [4].

In this work we revisit an ischemia detector developed by our group [5] and based on the root mean square (RMS) series of the repolarization interval. It was developed and validated using the ESCDB and here we incorporate new modules in order to improve its performance under the new LTSTDB perspective. We integrate a RMS based ST changes detector with a BPC detector to cancel out 


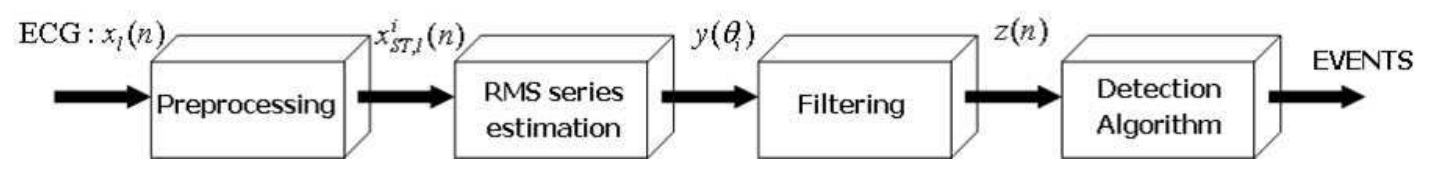

Figure 2. Block diagram of the ST changes detector presented in this work.

false alarms and improve the positive predictivity of the ischemia detector.

\section{Methods}

\section{1. $\quad$ Long Term ST Database}

The Long-Term ST Database consists of 86, two or three leads, 21 to 24 hour, Holter ECG recordings. Complete annotations have been provided for the database following different annotation protocols. One ST episode, ischaemic or non-ischaemic heart-rate related, had to be significant to be annotated according to the following rules: a) episode beginning when the magnitude of the ST deviation first exceeds $50 \mu V$, b) ending when the deviation becomes smaller than $50 \mu \mathrm{V}$, provided that it does not exceed 50 $\mu V$ in the following $30 \mathrm{~s}$ and $\mathrm{c}$ ) the deviation must reach a magnitude of $V_{\min }$ or more throughout a continuous interval of at least $T_{\min } \mathrm{s}$.

Three different protocols A, B and C are given depending on $V_{\min }$ and $T_{\min }$.

- Protocol A: $V_{\min }=75 \mu \mathrm{V}$ and $T_{\min }=30 \mathrm{~s}$.

- Protocol B: $V_{\min }=100 \mu \mathrm{V}$ and $T_{\min }=30 \mathrm{~s}$.

- Protocol C: $V_{\min }=100 \mu \mathrm{V}$ and $T_{\min }=60 \mathrm{~s}$.

\subsection{ST segment changes detector}

The RMS-based detector (see Figure 2) operates finding ST segment changes in the series resulting from computing the root mean square (RMS) difference series between the ST segment of a reference beat and the running beat. Then a threshold-based adaptive detector is applied on the series.

First, preprocessing techniques are applied on the raw ECG signal, $x_{l}(n)$, where $l$ is the corresponding lead and $n$ is the sample index. This preprocessing step consists of first applying a QRS detector [6] in order to find QRS fiductial points of each $i^{\text {th }}$ beat $\left(\theta_{i}\right)$ and selecting only normal beats, then baseline wander attenuation using cubic splines and finally rejection of noisy beats (those with differences in mean isoelectric level with respect to adjacent beats larger than $400 \mu \mathrm{V}$ ). In order to avoid the influence of high frequency noise in the RMS difference series, the ECG was low-pass filtered using a linear phase FIR filter with a cutoff frequency of $25 \mathrm{~Hz}$.

The ST segmentation (see Figure 1) is done selecting a fixed length window of $50 \mathrm{~ms}, x_{S T, l}^{i}(n)$, defined as:

$$
\begin{aligned}
& x_{S T, l}^{i}(n)=x_{l}\left(n_{S T_{0}}^{i}+n\right) \quad n=0, \ldots, N-1 \\
& \text { with } \quad n_{S T_{0}}^{i}=\theta_{i}+\frac{40}{1000} f_{s}+1.3 \sqrt{\frac{r r_{i}}{1000}} f_{s}
\end{aligned}
$$

where $N=\frac{50}{1000} f_{s}, f_{s}$ is the sampling frequency $(250 \mathrm{~Hz}$ in the whole database) and $r r_{i}$ represents the RR interval at the $i^{t h}$ beat in $m s$.

In order to calculate the RMS difference series, a reference beat, $\bar{x}_{S T, l}(n)$, has to be defined. We create a ST series defined as the first sample of the ST segment of each beat, $x_{S T, l}^{i}(1)$ with $i=1, \ldots, I$ where $I$ is the number of beats. On this series, an interval of 30 minutes called "basal interval" is searched with two restrictions: having the shorter peak to peak amplitude and being the whole interval series below $4 / 3$ of the median of the recording. Within this "basal interval" 100 beats are averaged to calculate the reference beat in each lead $\bar{x}_{S T, l}$.

Finally the RMS difference series, $y\left(\theta_{i}\right)$ is calculated using the following equation:

$$
y\left(\theta_{i}\right)=\sum_{l=1}^{L} \sqrt{\frac{1}{N} \sum_{n=0}^{N-1}\left(x_{S T, l}^{i}(n)-\bar{x}_{S T, l}(n)\right)^{2}}
$$

where $L$ is the number of leads. There are different factors such as motion artifacts that distort the RMS-series and therefore it needs post-processing. This is done by rejecting beats whose signal-to-noise ratio (SNR) differs more than $20 \mathrm{~dB}$ from the running exponentially averaged SNR series with constant factor equal to 0.02 . Latter, as outlier rejection, a median filter of length 5 beats is used on the RMS series. This series is evenly resampled to $1 \mathrm{~Hz}$ and an exponential averaged (with constant factor equal to 0.05) is applied to smooth the series resulting in a RMS- series suitable for analysis and denoted hereinafter by $z(n)$.

The final stage of the detection algorithm incorporates an adaptive threshold that works on the amplitude of the $z(n)$ series. This adaptation is included to account for the slow changing non-ischemic ST segment drift. The threshold adapts itself by adding a fixed amount " $\eta$ " to the baseline $\xi(n)$ of the $z(n)$ series, that is estimated as:

$$
\xi(n)=\xi(n-1)+\beta(z(n)-\xi(n-1))
$$

where $\beta$ is a parameter that adjusts the speed in the adaptation of the baseline to the $z(n)$ series. 


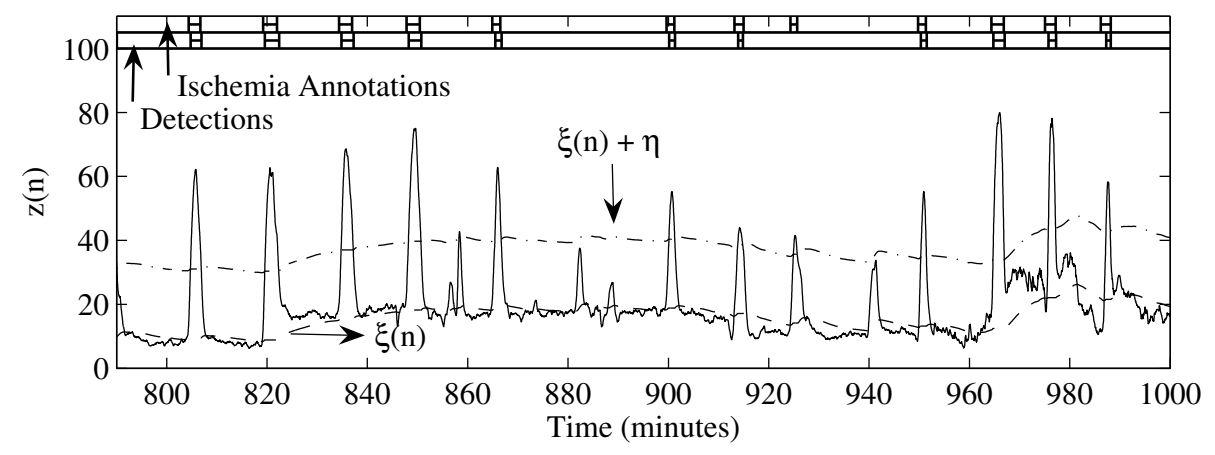

Figure 3. Example of ST changes detection on the record "s20591" showing, in the highest bar, the annotated ischemic events (protocol B) and in the lowest one the detections. The baseline estimation, $\xi(n)$, (dashed line) and the threshold (dotted-dashed line) are plotted over the $z(n)$ series.

When $z(n)>\xi(n)+\eta$ for a period of more than $45 \mathrm{~s}$, an event is detected. See Figure 3.

\subsection{BPC detector}

Body position changes are often manifested as shifts in the electrical axis and may be misclassified as ischemic changes during ambulatory monitoring. Previous studies use the Karhunen-Loève transform (KLT) to detect non ischemic episodes such as body position changes or conduction changes [7]. During these non ischemic events the QRS signatures change rapidly (generally over a period of half a minute) generating step function features in its KLT coefficient series.

In this work we modified a BPC detection technique [7] to account for conduction and axis shifts and then two series are used which are different combinations of pairs of series including the KLT or RMS from the QRS complex or ST segment. The detector first looks for step changes in both series, filtering them with a unit step function (216 $\mathrm{s}$ the two flat intervals with a linear transition in between of $72 \mathrm{~s}$ ) and applying a fixed threshold. Then a "flatness test" which consists of forcing a flat interval before and after each corroborated BPC detection is performed. Finally a logic combination of detected events in both series will be done resulting the final BPC detections.

The pair of series with better results was the RMS series of the QRS complex and the ST segment and therefore they were used in the final BPC detector.

\section{Results}

The performance of the detector is computed by two types of statistics: the gross $(g)$ and the average $(a)$ statistics [8]. In the gross statistics each episode has the same weight while in the average statistics the same weight is given to each record. Since the detector looks for changes in the ST-segment, ischemic and heart rate related events are combined in the sense of logical $O R$ fuction in the performance analysis. The annotation protocol B from the LTSTDB is used by default since it follows the same criteria as the ESCDB annotations in which the performance figures reached in terms of sensitivity / positive predictivity $(\mathrm{S} /+\mathrm{P})$ were $85 \% / 86 \%$.

First, point out that just a straight forward adaptation of the original RMS-based detector [5] tuned in the ESCDB with the parameter values, $\eta=18.1$ and $\beta=0.0083$, reduces its performance figures in the LTSTDB in terms of $S /+P$ to $70.5 \% / 68.2 \%$. See Table 1, "Original $R M S$ "

After including in the preprocessing step the "basal interval" search to define the reference beat $\bar{x}_{S T}(n)$, and using $\eta=21.5$ and $\beta=0.0103$, the performance improves in a significant way (S/P: $76.3 \% / 69.5 \%)$ as it is shown in Table 1, "No BPC det.".

An evaluation of the need of including BPC cancellation techniques gave a positive answer since an ideal BPC detector would improve the positive predictivity about $5 \%$. See Table 1, "Ideal BPC det."

Table 1. Performance of the original $(\eta=18.1, \beta=$ $0.0083)$ and modified ( $\eta=21.5, \beta=0.0103)$ RMSbased detectors on the LTSTDB. In the new RMS detector first the basal interval was included with no BPC detection, then it was used an ideal BPC detector, then our real BPC detector and finally we were restricted to detect only ischemic events and not HR events.

\begin{tabular}{|c|c|c|c|c|}
\hline & $\mathbf{S}(g)$ & $\mathbf{P}(g)$ & $\mathbf{S}(a)$ & $\mathbf{P}(a)$ \\
\hline \multicolumn{5}{|c|}{ ORIGINAL RMS } \\
\hline Original $R M S$ & $70.5 \%$ & $68.2 \%$ & $70.1 \%$ & $62.0 \%$ \\
\hline \multicolumn{5}{|c|}{ MODIFIED RMS WITH BASAL INTERVAL SEARCH } \\
\hline No BPC det. & $76.3 \%$ & $69.5 \%$ & $79.1 \%$ & $64.4 \%$ \\
\hline Ideal BPC det. & $76.3 \%$ & 74.7 & 79.1 & $70.1 \%$ \\
\hline Real BPC det. & $75.3 \%$ & $71.0 \%$ & $78.1^{c}$ & $66.4 \%$ \\
\hline No HR events & $79.6 \%$ & $62.2 \%$ & $82.0 \%$ & $52.4 \%$ \\
\hline
\end{tabular}


Using the developed BPC detector with the pair of series RMS from the QRS complex and RMS from the ST segment, the performance did not improve remarkably since the false BPC detections also reduce the $S$ of the ST changes detector. See Table 1, "Real BPC det."

If we are restricted to detect ischemia episodes and we just consider the heart rate (HR) related ones as false positives, the detector performance increases in $S$ ( see Table 1 "No HR events") but decreases in $+P$. Then showing the detector as suitable for ischemic events but claiming for appropriate cancelation rules to increase $+P$.

The performance analysis of the complete detector including the BPC cancellation detector, following the three different protocols, is shown in Table 2 with fixed parameters values $\eta=21.5$ and $\beta=0.0103$.

Table 2. Performance of the RMS-based detector on the LTSTDB with the real BPC detector in the three protocols.

\begin{tabular}{lcccc}
\hline \hline & $\mathbf{S}(g)$ & $\mathbf{P}(g)$ & $\mathbf{S}(a)$ & $\mathbf{P}(a)$ \\
\hline Protocol A & $56.3 \%$ & $82.8 \%$ & $61.7 \%$ & $80.5 \%$ \\
\hline Protocol B & $75.3 \%$ & $71.0 \%$ & $78.1 \%$ & $66.4 \%$ \\
\hline Protocol C & $84.1 \%$ & $60.5 \%$ & $83.7 \%$ & $54.4 \%$ \\
\hline \hline
\end{tabular}

\section{Discussion and conclusions}

The RMS-based detector showed a significant drop in performance on the LTSTDB compared to the results on the ESCDB. The main reason is due to the fact that the LTSTDB is much diverse then contains more data since it was developed to contain a wide variety of real-world data and therefore a greater number of non-ischaemic events compared to the ESCDB.

In the LTSTDB several combinations of different leads were used as they are appearing in the clinical praxis. This fact affects the detector performance negatively since ischemia is reflected differently in different leads.

The basal interval search gives remarkably improvements in the performance analysis (Table 1, "No BPC det.") while the BPC detector slightly improves the results (Table 1, "Real BPC det."). Nevertheless, the improvement reached the $20 \%$ of the maximum improvement (Table 1, "Ideal BPC det.") which evidence the difficulties in developing a robust BPC detector in ambulatory recordings.

If we try to just detect ischemic events avoiding heart rate related changes the sensitivity increases up to $82 \%$ (see Table 1, "No HR events"). This result suggests the need for the development of cancellation rules for this heart related changes, if those are not of interest.

If we observe the Table 2, the protocol $\mathrm{C}$ is the most restrictive, having then less annotated ischemic and heart rate related events what makes the detector improve the sensitivity. On the other hand, the protocol A is the less restrictive having many annotated episodes and making the sensitivity drop but improving in specificity.

In summary we certify that RMS based ischemia detector, tuned in the LTSTDB, decreases the performance obtained in the ESCDB and claims for much more robust false alarm cancellation rules, accounting for the very different nature of ST episodes.

\section{Acknowledgements}

This work was supported by the projects TEC2004-05263-C0202/TCM from MCYT/FEDER and T30 from DGA and also by a personal grant to A Mincholé of the program 'Europa XXI' patronized by CAI and CONSI+D.

\section{References}

[1] Jager F, Taddei A, Moody G, Emdin M, Antolič G, Dorn R, Smrdel A, Marchesi C, Mark R. Long-term st database: a reference for the development and evaluation of automated ischaemia detectors and for the study of the dynamics of myocardial ischaemia. Med Biol Eng Comput 2003;41:172182.

[2] Taddei A, Distante G, Emdin M, Pisani P, Moody G, Zeelenberg C, Marchesi C. The european st-t database: standard for evaluating systems for the analysis of st-t changes in ambulatory electrocardiography. Eur Heart J 1992;13:1164-1172.

[3] Jager F, Moody G, Marc R. Characterization of transient ischemic and non-ischemic st segment changes. In Comput. Cardiol. Los Alamitos: IEEE Comput. Soc. Press, 1995; 721-724.

[4] Smrdel A, Jager F. Automated detection of transient stsegment episodes in $24 \mathrm{~h}$ electrocardiograms. Med Biol Eng Comput 2004;42:303-311.

[5] Garca J, Srnmo L, Olmos S, Laguna P. Automatic deteccion of st-t complex changes on the ecg using filtered rms difference series: Application to ambulatory ischemia monitoring. IEEE Trans on Biomedical Engineering 2000;47(9):11951201.

[6] Moody G, Mark R. Development and evaluation of a 2lead ecg analysis program. In Computers in Cardiology. Los Alamitos: IEEE Comput. Soc. Press, 1982; 39-44.

[7] Garca J, Astrom M, Mendive J, Laguna P, L.Srnmo. Ecgbased detection of body position changes in ischemia monitoring. IEEE Trans Biomed Eng 2003;25(6):501-507.

[8] Jager F, Moody GB, Taddei A, Mark RG. Performance measures for algorithms to detect transient ischemic st segment changes. In Computers in Cardiology 1991. Los Alamitos: IEEE Computer Society Press, 1991; 369-372.

Address for correspondence:

Ana Mincholé

Departamento de Ingeniería Eléctrica y Comunicaciones

Maria de Luna 1, 50018, Zaragoza, Spain

E-mail address: minchole@unizar.es 